\title{
A STUDY OF COMMONLY USED CONVENTIONAL METHODS FOR GAS RESERVE ESTIMATION
}

\author{
Mohammad Shahedul Hossain and Mohammad Asad Ibne Hossain* \\ Department of Petroleum \& Mining Engineering, Shahjalal University of Science and \\ Technology, Sylhet-3114, Bangladesh
}

\begin{abstract}
Engr. Salma Akhter
Department of Chemical Engineering and Polymer Science, Shahjalal University of Science and Technology, Sylhet 3114, Bangladesh
\end{abstract}

Reserve estimation is very crucial in order to gain the confidence on profitable extraction of gas from a gas reservoir. Because the degree of uncertainty is inherent in the assessment, the reserves are 'estimated' rather than 'measured'. It is a dynamic process; reserves are updated and likely to change as more data are available in the course of the development of a gas field. Volumetric, Material Balance and Traditional Decline curve analysis are commonly used methods to estimate the reservoirs. After discovery, during initial delineation, volumetric method is used. When adequate production data are available, material balance is used to estimate the reserve. As depletion proceeds decline curve analysis is used to determine the remnant amount of reserve. However, modern estimation practice also uses type curves such as Blasingame, Fetkovich, Agarwal-Gardner as important tool to get a lucid idea about the reserve. They provide a more clear signal from the production data and quite handy to use and understand.

Keywords: reserve definition, volumetric method, material balance method, decline curve analysis, type curve.

\section{INTRODUCTION}

Natural gas is the naturally occurring hydrocarbon gas predominantly composed of methane. It is found in underground traps formed by structural and stratigraphic features called as reservoir. The prospect of accumulation of natural gas depends on coexistence of several factors in the subsurface which includes a) source rock, b) reservoir rock, c) trap, d) seal or cap rock etc. Once a reservoir is discovered, it is necessary to estimate the reserve of it. Reserve is the quantity of gas that is recoverable from the underground gas deposits. In many places natural gas and crude oil are found coexisting because their origin and accumulation processes are similar in many accounts. Ws shall discuss about the estimation process of dry gas reservoir as they are common in Bangladesh.

In 1986, the Society of Petroleum Engineers (SPE) adopted the following definition for reserves :

"Reserves are estimated volumes of crude oil, condensate, natural gas, natural gas liquids and associated marketable substances anticipated to be commercially recoverable and marketable from a given date forward, under existing economic conditions, by established operating practice, and under current government regulations."

The process of estimating oil and gas reserves for a producing field continues throughout the life of the field. There is always uncertainty in making such estimates. The level of uncertainty is affected by the following factors:

a) Reservoir type

b) Source of reservoir energy,

c) Quantity and quality of the geological, engineering, and geophysical data,

d) Assumptions adopted when making the estimate,

e) Available technology, and

f) Experience and knowledge of the evaluator.

Due to the degree of uncertainty is inherent in the assessment, the gas reserves are 'estimated' rather than 'measured'. Reserves are defined in different categories on the basis of uncertainty such as: 
a) Proved Reserve: up to $10 \%$ uncertainty

b) Probable Reserve: 10 to $50 \%$ uncertainty.

c) Possible Reserve: uncertainty level is as high as $90 \%$.

\section{CONVENTIONAL METHODS}

Different methods can be used to estimate the reserve depending on the maturity of the reservoir. The commonly used methods are:

a) Volumetric Method: at early stages.

b) Material Balance Method: when production data is available.

c) Decline Curve Analysis: when the reservoir reaches decline mode.

\subsection{Volumetric Method}

In the early stages of development, reserves estimates are restricted to the volumetric calculations. The volumetric method entails determining the physical size of the reservoir, the pore volume within the rock matrix, and the fluid content within the void space. This provides an estimate of the hydrocarbons-in-place, from which ultimate recovery can be estimated by using an appropriate recovery factor.

For a gas reservoir:

$$
G I I P=\frac{7758 \quad V_{R} \quad \varphi \quad\left(1-S_{W i}\right)}{B_{g i}} \times R . F
$$

Where:

$$
\begin{array}{ll}
\text { GIIP } & =\text { Gas initially in place }(\mathrm{SCF}) \\
V_{R} & =\text { Reservoir volume (acre-ft) } \\
\varphi & =\text { Porosity (fraction) } \\
\begin{array}{ll}
S_{W i} & =\text { Interstitial water saturation (fraction) }
\end{array} \\
\begin{array}{l}
B_{g i} \quad=\text { Initial gas formation volume factor } \\
(\text { res bbl/SCF) }
\end{array}
\end{array}
$$

R.F = Recovery factor (fraction)
2.1.1 Volume: The reservoir volume is obtained from geologic and fluid pressure analysis data. The geologist provides contour maps of the top and base of the reservoir, as shown in Figure: 1.

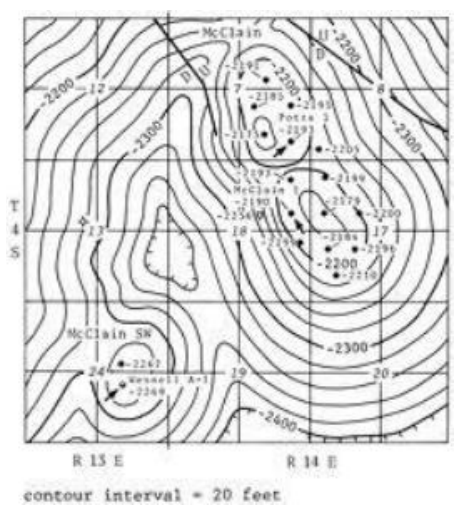

Figure 1: Contour Map

Such maps have contour lines drawn for every 20 feet, or so, the elevation can be determined by determining the gas water contact (GOC).

2.1.2 Porosity, $\varphi$ : Effective porosity of a sample is defined as the following ratio:

Effective Porosity $=\frac{\text { Interconnected } \text { pore volume }}{\text { Bulk volume }}$

Effective porosity of the reservoir rock can be determined from study of core analysis. The porosity of clean sandstones may be calculated from electric logs.

2.1.3 Interstitial water saturation, $S_{W i}$ : Water saturation is defined as the following ratio:

Water saturation $=\frac{\text { Volume of water present in pore spaces }}{\text { Volume of total pore spaces }}$

Interstitial or connate water saturation may be determined from electric logging information or by laboratory determinations run on cores by restored state, evaporation mercury injection, or centrifuge methods.

2.1.4 Formation volume factor , $\boldsymbol{B}_{\boldsymbol{g}}$,: Gas formation volume factor is the ratio of volume of gas at the reservoir condition to that of gas at the standard condition. Mathematically: 


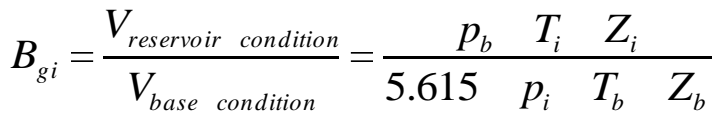

Where:

$B_{g i}=$ Initial gas formation volume factor (res $\mathrm{bbl} / \mathrm{SCF}$ )

$p_{b}=$ Base pressure (psia)

$p_{i}=$ Initial reservoir pressure (psia)

$T_{b}=$ Temperature at base conditions $\left({ }^{0} \mathrm{R}\right)$

$T_{i}=$ Temperature at initial conditions $\left({ }^{0} \mathrm{R}\right)$

$Z_{b}=$ Gas deviation factor at base conditions

$Z_{i}=$ Gas deviation factor at initial reservoir conditions.

2.1.5 Recovery factor ( R.F) : Recovery factor is a number between zero and unity representing the fraction of recoverable oil. It depends on the following factors:

a) Current economic circumstances.

b) Environmental and ecological considerations.

c) Governmental regulations as well as politics.

d) Physics of the reservoir-fluid system.

Finally, GIIP can be obtained by using all the parameters in the initial equation.

Although volumetric method is the only method available at the early stage of the reservoir, the result might be harmed by erroneous determination of the reservoir area. Reservoir boundary is subject to large errors. Again, this method cannot provide the EUR (Expected Ultimate Recovery).

\subsection{Material Balance Method}

Material balance methods provide a simple, but effective alternative to volumetric methods for estimating not only original gas in place, but also gas reserve at any stage of reservoir depletion. Material balance equation is simply a statement of conservation of mass, or

(Original hydrocarbon mass)-(Produced hydrocarbon mass $)=($ Remaining hydrocarbon mass)

The general material balance equation reduces to the conventional gas material balance equation as :
$G\left[B_{g}-B_{g i}\left\{1-\frac{\left(c_{w} S_{w i}+c_{f}\right)\left(p_{i}-p\right)}{1-S_{w i}}\right\}\right]+5.615 \quad W_{e}=\bar{G}_{p} B_{g}+5.615 \quad \bar{W}_{p} B_{w}$

This equation can be reduced to a simplified form based on a few assumptions.

2.2.1 Without Water Influx : If there is no external energy from other sources, such as aquifer is applied, the reservoir is considered as completely enclosed. If $\mathrm{Wp}=0 ; \mathrm{W}_{\mathrm{e}}=0$ and $\mathrm{HCPV}=$ constant, then the equation (3) reduces to:

$$
\frac{p}{Z}=\left[\frac{p_{i}}{Z_{i}}-\frac{p_{i}}{Z_{i}} \frac{G_{p}}{G}\right]
$$

This equation is applicable for:

1. Constant reservoir temperature

2. No phase change in the reservoir

3. No water influx

4. No rock compaction

5. No connate water expansion.

From equation (4) a plot of $p / Z$ vs. $G_{p}$ can be found.

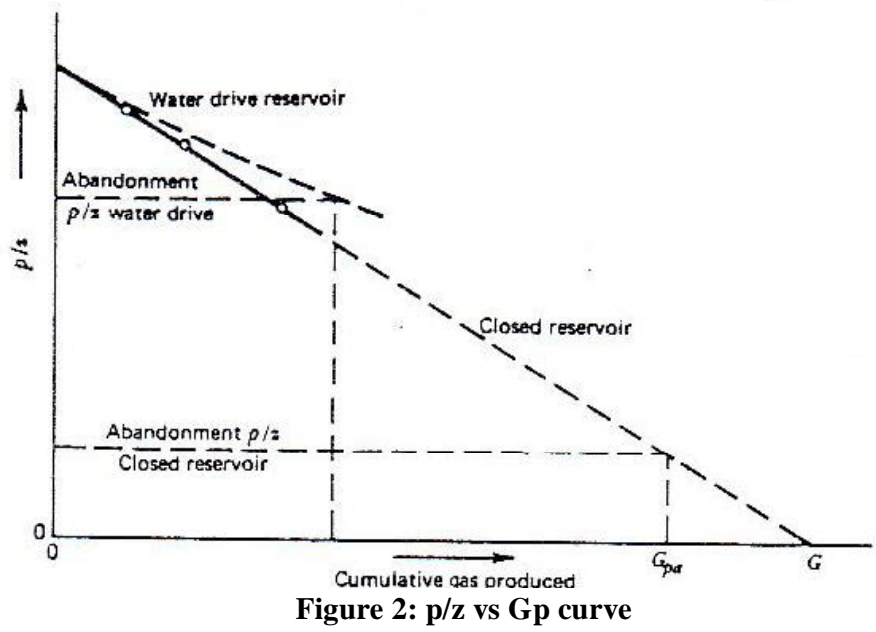

\subsubsection{With water influx}

If there is a water drive in the reservoir, then the pore volume is reduced by the amount equal to the volume of encroaching water. Now, considering water influx, equation (3) becomes:

$$
G B_{g i}=\left(G-G_{p}\right) B_{g}+W_{e}-W_{p} B_{w} . .(5)
$$

Now , using production data we can use equation (4) or (5) to determine the amount of gas remaining $\left(\mathrm{G}-G_{p}\right)$ in the reservoir. 
Material balance is a good tool to measure the reserve. But, for material balance it is needed to shut down the reservoir to determine the parameters. Thus, the data is as available as production data and the well manager is unwilling do it frequently as it not economically feasible.

\subsection{Decline Curve Analysis}

The production rate of wells, or groups of wells, generally declines with time. An empirical formula can sometimes be found that fits the observed data so well that it seems rather safe to use the formula to estimate future relationships.

In most case the production will decline at a decreasing rate, that is, $\mathrm{dq} / \mathrm{dt}$ will decrease with time. The figure below shows an identical curve. The $\mathrm{t}=0$ point can be chosen arbitrarily. $\mathrm{q}$ is the gas production rate and $t$ is time. The area under the curve between the times $t_{1}$ and $t_{2}$ is a is a measure of the cumulative production during this time period since

$$
G_{p}=\int_{t_{1}}^{t_{2}} q d t
$$

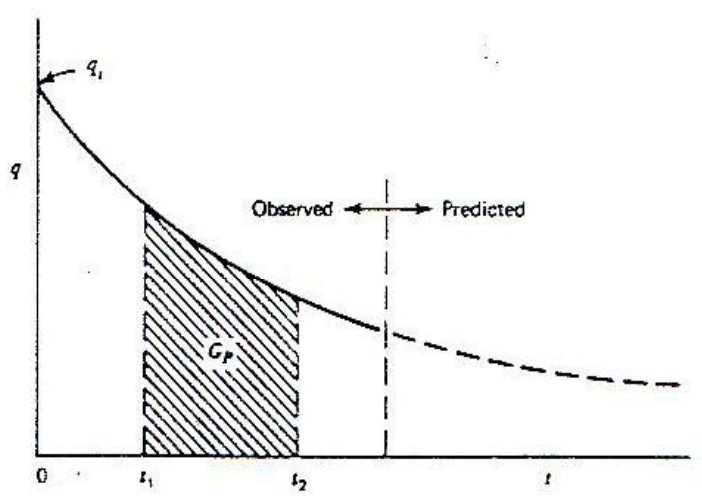

Figure 3: production rate vs time

There are three commonly recognized types of decline curves. Each of these has a separate mathematical form that is related to the second factor, which characterizes a decline curve, that is, the curvature. These types are referred to as:

1. Constant-percentage or exponential decline.

2. Hyperbolic decline.

\section{Harmonic decline.}

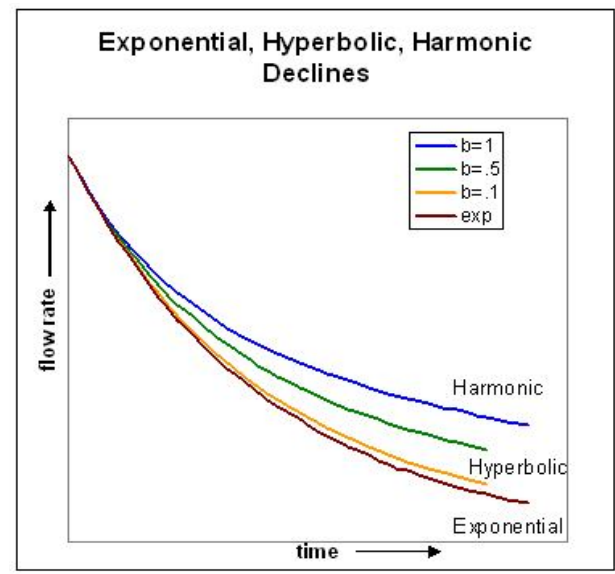

Figure 4 Exponential, Hyperbolic and Harmonic Decline Curve

\subsubsection{Exponential Decline:}

In the exponential decline, the well's production data plots as a straight line on a semilog paper. The equation of the straight line on the semilog paper is given by:

$q=q_{i} e^{-D t}$

Where:

$q=$ well's production rate at time $\mathrm{t}, \mathrm{SCF} / \mathrm{day}$

$\mathrm{qi}=$ well's production rate at time $0, \mathrm{SCF} /$ day

$\mathrm{D}=$ nominal exponential decline rate, $1 /$ day

$\mathrm{t}=$ time, day

\subsubsection{Hyperbolic Decline:}

Alternatively, if the well's production data plotted on a semilog paper concaves upward, then it is modeled with a hyperbolic decline. The equation of the hyperbolic decline is given by:

$$
q=q_{i}\left(1+b D_{i} t\right)^{-1 / b}
$$

Where:

$\mathbf{q}=$ well's production rate at time $\mathrm{t}, \mathrm{SCF} /$ day

$q \mathrm{i}=$ well's production rate at time $0, \mathrm{SCF} /$ day

$\mathrm{Di}=$ initial nominal exponential decline rate $(\mathrm{t}=$ $0), 1 /$ day

$\mathrm{b}=$ hyperbolic exponent

$\mathrm{t}=$ time, day

\subsubsection{Harmonic Decline :}

A special case of the hyperbolic decline is known as "harmonic decline", where b is taken to be equal to 1 . 


$$
q=q_{i}\left(1+D_{i} t\right)^{-1}
$$

Where:

$q=$ well's production rate at time $t, S C F /$ day

$\mathrm{q} i=$ well's production rate at time $0, \mathrm{SCF} / \mathrm{day}$

$\mathrm{Di}=$ initial nominal exponential decline rate $(\mathrm{t}=$

$0), 1 /$ day

$\mathrm{b}=$ hyperbolic exponent $=1$

$\mathrm{t}=$ time, day

Decline curve relationships are empirical, and rely on uniform, lengthy production periods.

Decline curves are the most common means of forecasting production. They have many advantages such as:
a) Data is easy to obtain,
b) They are easy to plot,
c) They yield results on a time basis, and
d) They are easy to analyze

In decline curve analysis, usually the flowing pressure is assumed to be constant as the production rate declines. But in the real conditions, wellhead pressure also varies with time. So, the reserve estimated by decline curve analysis often varies from actual reserve.

\section{MODERN TYPE CURVE METHODS:}

Type curve matching is essentially a graphical technique for visual matching of production data using preplotted curves on a log-log paper.

From matching of field data with pre plotted data, dimensionless reservoir radius is determined, which is then used to calculate the reserve.

Type curves are also used as a valuable diagnostic tool. It provides a practical interpretation of data to qualitative conclusion about well production. For example we can explore the following sample type curve.

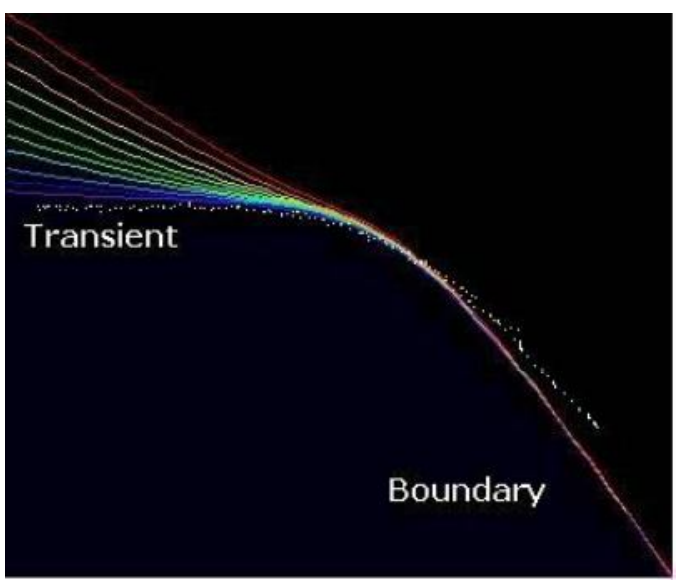

Figure 5: Type Curve as a Diagonstic Tool

It is evident that at the early stage the production data do not match with the type curve and the trend indicates a damaged reservoir. Then the decline indicates the boundary effect. At the late stage the raise of the curve indicates the presence of pressure support. The support might be from water drive, partially sealing fault, multi layer effect, communicating gas reservoirs etc. If the reservoir response line was lower than pre plotted decline line, it would have been an indication of liquid loading effect.

Modern reserve estimation practice is complemented by various type curves. Several type curves for the same reservoir are used to confirm the reserve. Type curve analysis are done from production data (flowing pressure, flow rate) which are available in the producing reservoirs. Three major Type Curve Analysis methods are described as below:

\subsection{Fetkovich Analysis}

Fetkovich was the first to extend the concept of using type curves to transient production. The Fetkovich methodology uses the depletion for the analysis of boundary-dominated flow and constant pressure typecurves (originally developed by VanEverdingen and Hurst) for transient production. The most valuable feature of typecurves lies not in the analysis, but in the diagnostics.

There are two sets of curves that converge in the centre. Matching data on the left side provides information about the transient behaviour of the system $(\mathrm{k}, \mathrm{s})$ while the right side gives 
information about the boundary dominated behavior of the reservoir (OGIP, Area).

The left-hand side of the Fetkovich type curves ais derived from the analytical solution to the flow of a well in the centre of a finite circular reservoir producing at a constant wellbore flowing pressure. Fetkovich showed that, for all sizes of reservoirs, when transient flow ended, the boundary-dominated flow could be represented by an exponential decline.

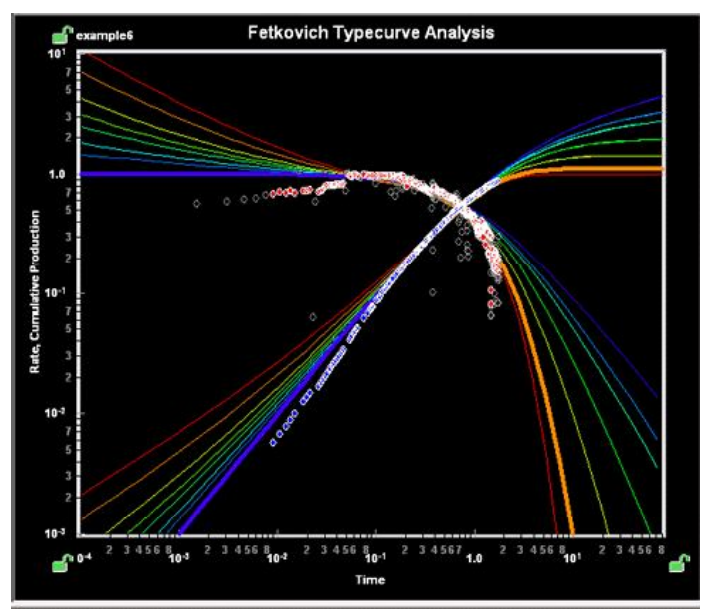

Figure 6: Sample Fetkovich Type Curve

Real data set can't be matched with the curve properly, because Fetkovich analysis assumes that production occurs under constant bottomhole flowing pressure conditions.This is the major source of error for Fetkovich analysis.

\subsection{Blasingame Analysis}

The production decline analysis techniques of Fetkovich is limited in that it does not account for variations in bottomhole flowing pressure in the transient regime, and only account for such variations empirically during boundary dominated flow. In addition, changing PVT properties with reservoir pressure are not considered for gas wells.

Blasingame typecurve uses a form of superposition time function that only requires one depletion stem for typecurve matching; the harmonic stem. One important advantage of this method is the typecurves used for matching are identical to those used for Fetkovich decline analysis, without the empirical depletion stems. When the typecurves are plotted using Blasingame's superposition time function, the analytical exponential stem of the Fetkovich typecurve becomes harmonic. The significance of this may not be readily evident until considering that, if the inverse of the flowing pressure is plotted against time, pseudo-steady state depletion at a constant flow rate follows a harmonic decline. In effect, Blasingame's typecurves allow depletion at a constant pressure to appear as if it were depletion at a constant flow rate. In fact, Blasingame et. al. have shown that boundary-dominated flow with both declining rates and pressures appear as pseudosteady state depletion at a constant rate, provided the rate and pressure decline monotonically.

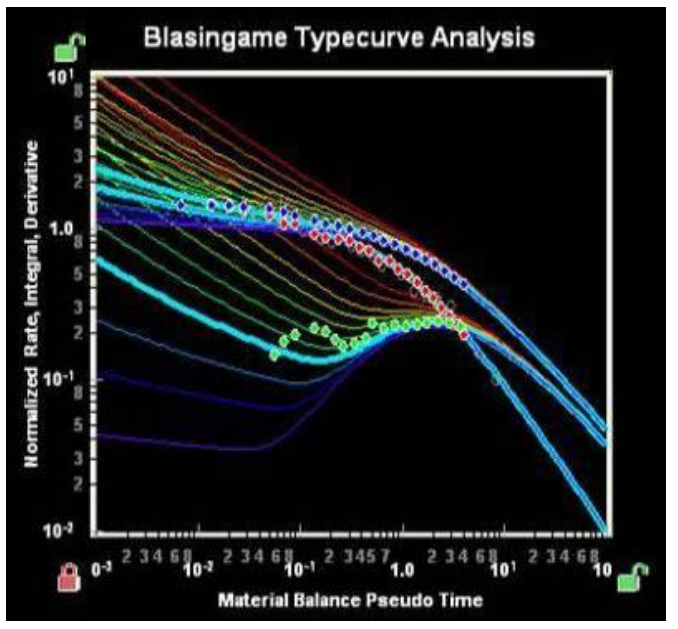

Figure 7: Blasingame Type Curve

Blasingame's improvements on the Fetkovich style of production decline analysis are further enhanced by the introduction of two additional typecurves which are plotted concurrently with the normalized rate typecurve. The 'rate integral' and 'rate integral derivative' typecurves aid in obtaining a more unique match.

The Flowing Material Balance method provides convenient and sensitive way to estimate Gas in place without shut-in pressure except for $P i$. This method has better resolution for boundary 
dominated flow than any of the production analysis methods.

A Blasingame type-curve plot can be displayed representing the response of a vertical well in a closed circle, with the data overlaid. This plot is also present for gas where material balance time is replaced by a material balance pseudo time. The loglog plot can be used as a diagnostic tool with exceptionally clean data although even with scattered data, trends may be detected. The simulated model can be compared to the data on both of these plots.

\subsection{Agarwal-Gardner Rate-Time Analysis:}

Agarwal and Gardner have compiled and presented new decline typecurves for analyzing production data. Their methods build upon the work of both Fetkovich and Blasingame, utilizing the concepts of the equivalence between constant rate and constant pressure solutions. Agarwal and Gardner present new typecurves with dimensionless variables based on the conventional welltest definitions, as opposed to the Fetkovich dimensionless definitions used by Blasingame et. al.. They also include primary and semi-log pressure derivative plots (in inverse format for decline analysis). Furthermore, they present their decline curves in additional formats to the standard normalized rate vs. time plot. These include the rate vs. cumulative and cumulative vs. time analysis plots.

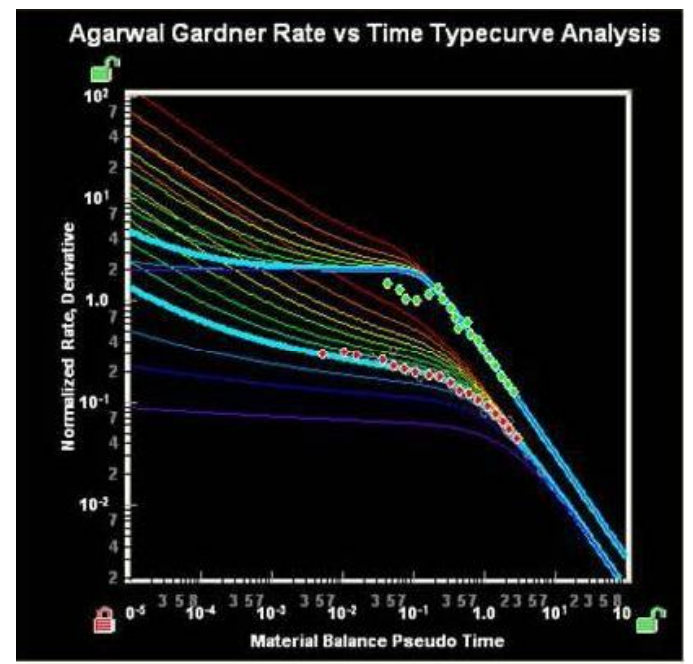

Figure 8: Agarwal Gardner Rate vs Time Typecurve Analysis
The Agarwal-Gardner Rate-Time analysis plot includes dimensionless typecurves, based on the constant rate solution. Unlike Blasingame, these typecurves are graphed using the welltest dimensionless variables $\mathrm{q}_{\mathrm{D}}$ and $\mathrm{t}_{\mathrm{DA}}$. Agarwal and Gardner also include typecurves for the primary pressure derivative and semi-logarithmic (welltest) derivative (plotted as an inverse). The result is a diagnostic analysis that clearly shows the transition point from transient to boundary dominated flow.

\section{CONCLUSION}

Reserve estimation is very crucial for a reserve to make decisions. Estimation provides the confidence about economical feasibility to produce from a reservoir; whether it is cost worthy or not. In this paper a few conventional and type curve methods are discussed. Other methods like analogy, reservoir simulation are also used to estimate the reserve. Unfortunately none of them is foolproof. It is thus recommended to use several methods at a time with consideration of their merits and drawbacks. The final reserve should be declared by averaging the valid output of those methods. It should be noted that the total amount reserve may vary with respect to time due to accumulation or migration of gas. So reserve estimation should be done in a regular basis to have clear idea on current reserve.

\section{REFERENCES}

1. Lee, J. and Wattenbargar, R.A.(1996), Gas Reserve Estimation, SPE Textbook Series, Volume 5, pp. 230-235

2. Imam, B.(2005), Energy Resources of Bangladesh, University Grants Commission of Bangladesh, pp.62-66

3. Dake, L.P.(1991), Fundamentals of reservoir engineering(twelfth edition), Elsevier,,Amsterdam.

4. Craft, B.C. and Hawkins, M.F.(1991),Applied Petroleum Reservoir Engineering (second Edition),Prentice Hall, N.J

5. Selley, R.C., Elements of Petroleum Geology(second edition), Academic Press, San Diego. pp. 291-297 
6. William, D. and Mccain, Jr., Petroleum Fluids(second Edition), Pennwell books, Tulsa,Oklahoma

7. Gaskari, R., Mohaghegh, S. D. and Jalali, J., New Method for Production Data Analysis to Identify New Opportunities in Mature Fields: Methodology and Application.

8. Blasingame, T.A; McCray, T.L; Lee, W.J: "Decline Curve Analysis for Variable Pressure Drop/Variable Flowrate Systems," paper SPE 21513 presented at the SPE Gas Technology Symposium, 23-24 January, 1991.

9. Agarwal, R.G; Gardner, D.C;

Kleinsteiber, S.W; and Fussell, D.D.:

"Analyzing Well Production Data Using Combined Type Curve and Decline Curve Analysis Concepts," paper SPE 57916 presented at the $1998 \mathrm{SPE}$ Annual Technical Conference and Exhibition, New Orleans, 27-30 September.

10. Fetkovich, M.J.: "Decline Curve using Type Curves," JPT (June 1980), 1065

11. Fetkovich, M.J; Fetkovich, E.J; and Fetkovich, M.D.: "Useful Concepts for Decline Curve Forecasting, Reserve Estimation, and Analysis," SPERE (February 1996), 13. 